\title{
Optimal reactive power compensation using synchronous generators
}

\author{
Constantin GHIȚA $\breve{A}^{1}$, Aurelian CRĂCIUNESCU², Valentin NĂVRĂPESCU ${ }^{3}$, Ioan Dragoş DEACONU ${ }^{4}$, Aurel - \\ Ionuț CHIRILA $\breve{A}^{5}$, Ion - Daniel ILINA ${ }^{6}$ \\ 1,2,3,4,5,6 Electrical Machines and Drives Department \\ Electrical Engineering Faculty, Universitatea Politehnica Bucuresti \\ Splaiul Independenței 313 (Romania) \\ e-mail: ghita.constantin@gmail.com
}

\begin{abstract}
The paper presents a study on synchronous machine that can operate as a generator and a compensator simultaneously. Modifying the field current, the reactive power injected into the supplying network is changed. An automated system is described which injects the maximum reactive power for a given amount of electrical active power, under the following conditions: the stator current is limited to the rated value, the field current is maximum admissible, the grid voltage is limited to a maximum reference and over-excited state of the machine is assured.
\end{abstract}

\section{Key words}

Synchronous generator, Power factor, Active power, Reactive power

\section{Introduction}

The synchronous machine is more and more used for wind electrical generation systems. For increased powers the electrically excited type is employed. For medium and low power the permanent magnet synchronous machine is used [1] or hybrid excited type, i.e. both permanent magnet and electrically field generation systems are used in correlation. In cases of electrically excited generators, when the machine is over-excited it generates reactive power into the grid. For long lasting industrial applications, for safety reasons, the thermal requirements should be met. Whatever the situation the stator currents must not exceed the rated value and the field current should not overcome their maximum admissible values.

The synchronous generators inject active power into the grid, but can also inject reactive power in case they are over-compensated. The injected reactive power is controlled through the field current. The paper describes an automated system, attached to a synchronous generator, that has as objective to inject the maximum possible reactive power for any injected active power lower than the rated value, under the following conditions: the stator current is limited to the rated value, the field current is maximum admissible, the grid voltage is limited to a maximum set value and the over-excited synchronous generator operation is assured.

When neglecting the stator resistance, the vector diagrams corresponding to the over-excited state are shown in Fig. 1.a (for round rotor generators) and Fig. 1.b (for salient-pole generators) [2]-[4], where: $U, I$ are the voltage vector and the stator current vector respectively, $E_{\mathrm{o}}$ represents the back e.m.f. vector, $X_{\mathrm{s}}$ is the round rotor synchronous generator's reactance, $X_{\mathrm{d}}, X_{\mathrm{q}}$ are the salient-pole rotor synchronous generator's reactance, $\delta$ is the load angle and $\varphi$ represents the angle between the grid's voltage vector and the generator's statoric current vector.

Based on the vector diagrams presented in Fig. 1.a, the following equation can be written:

$$
E_{o}=\sqrt{\left(X_{s} I\right)^{2}+U^{2}+2 U X_{s} I \sin \varphi}
$$

Using the diagram shown in Fig. 1.b results:

$$
\begin{aligned}
& E_{o}=U \cos \delta+X_{d} I_{d} \\
& U \sin \delta=X_{q} I_{q}
\end{aligned}
$$

where $I_{\mathrm{d}}$ and $I_{\mathrm{q}}$ are the statoric current's components over the direct and quadrature axis of the generator, defined as:

$$
\begin{aligned}
& I_{q}=I \cos (\varphi+\delta) \\
& I_{d}=I \sin (\varphi+\delta)
\end{aligned}
$$

Replacing the currents from (3) in (2) yields:

$$
\begin{aligned}
& \left(X_{d} I \cos \varphi\right) \sin \delta+\left(X_{d} I \sin \varphi+U\right) \cos \delta=E_{o} \\
& \left(X_{q} I \sin \varphi+U\right) \sin \delta-\left(X_{q} I \cos \varphi\right) \cos \delta=0
\end{aligned}
$$

Using (4) the quantities sin $\delta$ and $\cos \delta$ are obtained. Then, summing the squares of these quantities and performing some calculations, the following relation is obtained: 


$$
\begin{aligned}
& U^{2}+X_{d} X_{q} I^{2}+U I\left(X_{d}+X_{q}\right) \sin \varphi= \\
& =E_{o} \sqrt{U^{2}+X_{q}^{2} I^{2}+2 U I X_{q} \sin \varphi}
\end{aligned}
$$

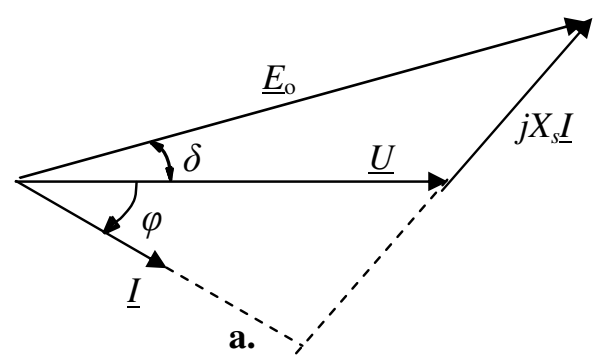

It is obvious that if in (5), that is valid for the salient-pole generator, the reactances are set accordingly to $X_{d}=X_{q}=X_{s}$, expression (1) is obtained, valid for the round-rotor generator.

Fig. 1. Vector diagrams corresponding to the over-excited synchronous generator: $\mathbf{a}$ - round rotor; $\mathbf{b}$ - salient-pole rotor.

\section{Maximum admissible field current for a given electrical active power}

When the generator delivers to the grid an amount of active power less than the rated one $\left(P<P_{\mathrm{r}}\right)$ it stills can compensate only a part of the required reactive power by increasing the DC field current, in the over-excited state $\left(I_{\mathrm{e}}>I_{e}^{*}\right)$. The maximum admissible value of DC field current is $I_{\mathrm{emax}}$ corresponding to the rated stator current. The value $I_{e}^{*}$ represents the optimal excitation when both the minimum stator current and maximum power factor $(\cos \varphi=1)$ are obtained. In other words, it represents the minimum point of the $\mathrm{V}$-curve $I=f\left(I_{\mathrm{e}}\right)$ for a given active power $P$.

For salient-pole rotor the following data of the synchronous generator are known: rated power $S_{r}$, rated phase voltage and current $U_{r}$ and $I_{r}$, synchronous reactance $X_{d}$ and $X_{q}$, magnetizing characteristic $E_{\mathrm{o}}=f\left(I_{e}\right)$. The generated reactive power and the phase angle between the voltage and the current are:

$Q=\sqrt{S_{r}^{2}-P^{2}}=3 U_{r} I_{r} \sin \varphi \Rightarrow \sin \varphi=\frac{\sqrt{S_{r}^{2}-P^{2}}}{3 U_{r} I_{r}}$

Replacing $\sin \varphi$ from (6) in (5) the maximum admissible field current yields:

$$
I_{e \max }=f^{-1}\left(\begin{array}{c}
\frac{U_{r}^{2}+X_{d} X_{q} I_{r}^{2}}{\sqrt{U_{r}^{2}+X_{q}^{2} I_{r}^{2}+2 X_{q} \cdot \frac{\sqrt{S_{r}^{2}-P^{2}}}{3}}}+ \\
+\frac{\left(X_{d}+X_{q}\right) \cdot \frac{\sqrt{S_{r}^{2}-P^{2}}}{3}}{\sqrt{U_{r}^{2}+X_{q}^{2} I_{r}^{2}+2 X_{q} \cdot \frac{\sqrt{S_{r}^{2}-P^{2}}}{3}}}
\end{array}\right)
$$

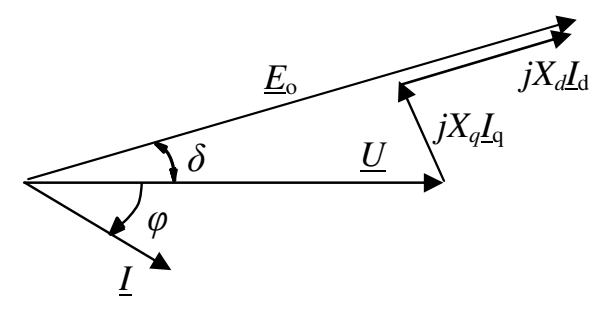

b. so that, the generator delivers to the grid the set active power $P$, and the statoric current is less than the rated value $I_{\mathrm{r}}\left(U_{\mathrm{r}}\right.$ represents the grid's voltage rated value).

For round rotor, $X_{\mathrm{d}}=X_{\mathrm{q}}=X_{\mathrm{s}}$, using (7) the maximum admissible field current is:

$$
I_{e \max }=f^{-1}\left(\sqrt{U_{r}^{2}+X_{s}^{2} I_{r}^{2}+2 X_{s} \cdot \frac{\sqrt{S_{r}^{2}-P^{2}}}{3}}\right)
$$

Equations (7) and (7') allow obtaining the maximum field current in the cases of salient-pole and round-rotor generators, corresponding to an over-excited state of the generator, when the injected active power into the grid is less than the rated value and the generator compensates the power factor of the grid where it is connected.

\section{Numerical example}

A round rotor synchronous generator the following data: $S_{\mathrm{r}}=400 \mathrm{kVA} ; U_{\mathrm{r}, \mathrm{ph}}=3470 \mathrm{~V} ; f=50 \mathrm{~Hz} ; X_{\mathrm{s}}=50 \Omega$; $R_{1}=0 ; \eta=1$ (losses are neglected). The no-load generator curve is given in Fig. 2 .

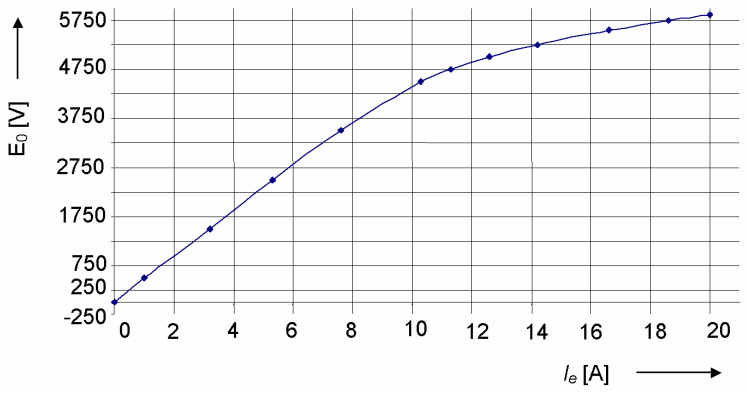

Fig. 2. The no-load curve of the synchronous generator 
The rated phase current:

$$
I_{r}=\frac{S_{r}}{3 U_{r, p h}}=\frac{400 \cdot 10^{3}}{3 \cdot 3470}=38.42 \mathrm{~A}
$$

The back e.m.f. $E_{o}^{*}$, corresponding to the optimal field current $I_{e}^{*}$ (when $\varphi=0$ ) in case of rated values of the current and the phase voltage, can be obtained from Fig. 1.a (right triangle $\underline{U}_{r}, j X_{s} \underline{I}_{r}, \underline{E}_{0}$ - when $\varphi=0$ ):

$$
E_{0}^{*}=\sqrt{U_{r}^{2}+\left(X_{s} I_{r}\right)^{2}}==\sqrt{3470^{2}+(50 \cdot 38.42)^{2}}=3966 \mathrm{~V}
$$

The optimal field current corresponding to this voltage is $I_{e}^{*}=9.05$ A (see Fig. 2).

Let us suppose that the generator must deliver to the grid an active power $P=200 \mathrm{~kW}$. Using (7') the maximum field current is:

$I_{e \max }=f^{-1}(5222)=14.1 \mathrm{~A}$

Table 1 shows the maximum admissible field current variation with the generator's load.

Table 1: The generator's maximum admissible field current and powers when $I=I_{r}$.

\begin{tabular}{|c|c|c|c|c|c|}
\hline$P[\mathrm{~kW}]$ & 400 & 300 & 200 & 100 & 0 \\
\hline$Q[\mathrm{kVAr}]$ & 0 & 265 & 346 & 387 & 400 \\
\hline$I_{\text {emax }}[\mathrm{A}]$ & 9.05 & 12.3 & 14.1 & 14.9 & 15.1 \\
\hline
\end{tabular}

As conclusion when stator current is constant (here rated value), the active power decreases, the reactive power injected into grid increases and the maximum admissible field current also increases. For rated active power the reactive power is null. For no load operation the reactive power is maximum and so is the field current (15.1 A). The maximum compensation for the rated stator current regardless the electric active power is obtained for a field current between $(9.05 \ldots 15.1) \mathrm{A}$.

\section{Reactive power compensation automated system}

The automated system has to fulfill the following conditions:

- regardless the delivered electric active power, the system generates the maximum reactive power by automatic adjustment of the field current;

- the stator current must not exceed the rated value;
- the generator is over-excited, that is $I_{e}>I_{e}^{*}$ regardless the delivered electric active power.

The generator can operate under-excited at rated current $\left(I_{e}<I_{e}^{*}\right)$ but it needs reactive power from the grid and so the grid's power factor is affected. To avoid this situation the optimal field current curve $I_{e}^{*}=g(P)$ is needed. If $100 \%$ efficiency is considered (losses are neglected) then the optimal field current characteristic can be extracted from (1) if voltage and current are considered in phase $(\varphi$ $=0)$.

Current $I$ can be expressed:

$$
I=\frac{P}{3 U_{r}}
$$

So:

$I_{e}^{*}=f^{-1}\left(E_{o}^{*}\right)=f^{-1}\left[\sqrt{\left(\frac{X_{s} P}{3 U_{r}}\right)^{2}+U_{r}^{2}}\right]=g(P)$

where $U_{\mathrm{r}}$ is the rated voltage. Table 2 presents the optimal field current $I_{e}^{*}=g(P)$

Table 2: The generator's optimal field current (for previous numerical example).

\begin{tabular}{|c|c|c|c|c|c|}
\hline$P[\mathrm{~kW}]$ & 0 & 100 & 200 & 300 & 400 \\
\hline$I_{e}^{*}[\mathrm{~A}]$ & 7.51 & 7.60 & 7.83 & 8.35 & 9.05 \\
\hline
\end{tabular}

In order for the system to compensate regardless the electric active power $P$, the field current must always be greater than values shown in Table 2 .

In addition to the previously presented conditions, the system must perform grid voltage regulation. Under no case it must exceed rated value especially for low power grids. This is achieved by limiting the field current [6].

The diagram of the automated system is presented in Fig.3. The main components are: current transducers (TC1, TC2), grid voltage transducer (TTR1), field's voltage transducer (TTR2), freewheel diode (DRL), switch transistor (IGBT), processing unit $(\mu \mathrm{P})$, singlephase bridge $(\mathrm{MB})$, filtering capacitor $\left(\mathrm{C}_{f}\right)$, and driver (D).

The schematic is designed for medium voltage grids $(6 \ldots 15 \mathrm{kV})$. In this case, a transformer should be added before (MB) to lower the voltage, (TTR1) is connected to the bridge input.

The maximum variation of the field current is obtained for generator's low loads when maximum compensation is requested. This variation should not influence the dynamic stability of the generator. I other words, the 
automated system should change the field current at a this current is small and has no influence over the dynamic stability of the generator.

The transducer (TTR1) reads the transmitted supplying grid voltage to the microprocessor. The transducer low rate. If the field current is not constant the ripple of (TTR2) monitors the rectified voltage given supplied by the MB bridge, and the (D) driver controls and fully protects the IGBT (i.e. overloads, temperature, shortcircuits) [7]

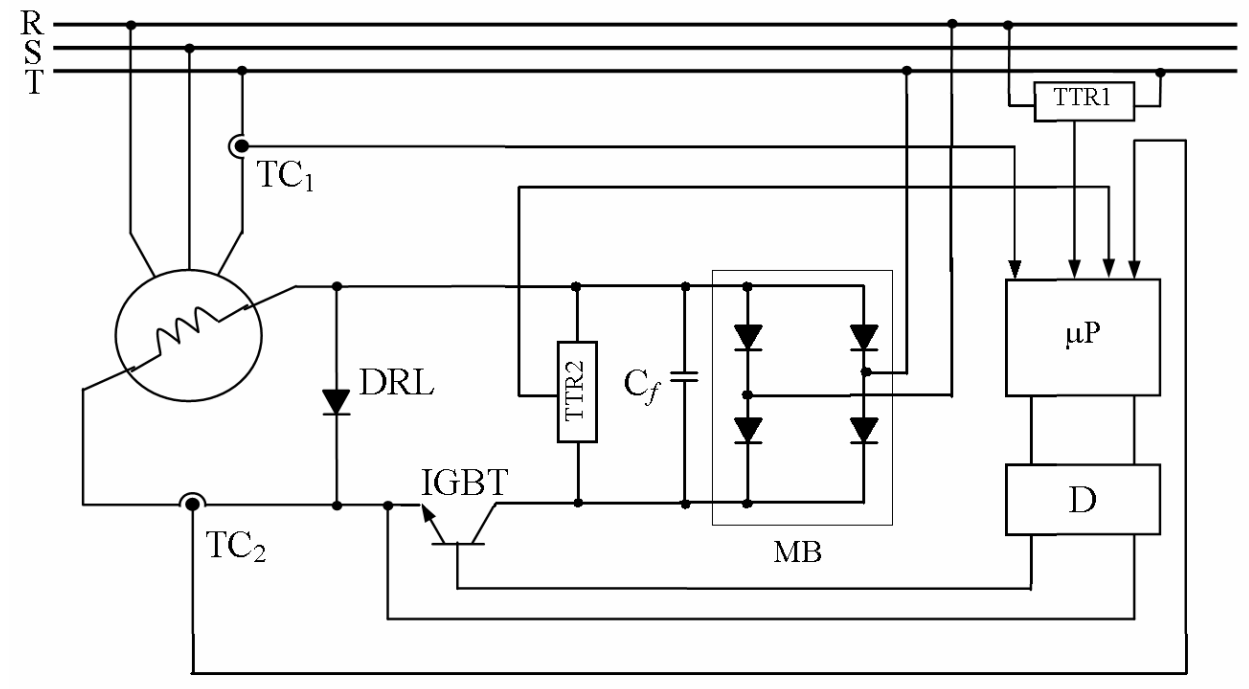

Fig. 3. Automated system diagram.

\section{Conclusion}

The synchronous machine can simultaneously operate as a generator and a compensator. Changing the field current, the reactive power exchanged with the grid is modified. The paper deals with the over-excited synchronous generator. Thus, for a given load (active power $P$ ) and a stator current less than the rated value, the maximum field current can be deducted so that the maximum reactive power is achieved.

The presented regulation system has a simple configuration and it is easy to be manufactured due to its cheap and safe components. It performs the following tasks: the stator current is limited to the rated value, the field current is maximum allowed, the grid voltage is limited to a set value and the over-excited state of the machine is assured regardless the active power load.

This design can also be used for greater powers for low and medium voltage generators. In case of medium voltage it is recommended to use a transformer before the rectifier to lower the voltage in accordance with grid voltage and excitation voltage. The time response is small and it depends on the excitation circuit inductance.

The synchronous generators are used for wind energy generation systems (local grids) but also for classical power generation stations that deliver the energy for national grids. For these generators, the described system is very useful. Moreover, the implementing cost is low due to the schematics simplicity.

\section{References}

[1] R. Noroozian, M. Abedi ,G.B. Gharehpetian, S.H..Hosseini,

"Modeling and simulation of the microturbine generation system for on-grid and off-grid operation mode" in Proc. ICREPQ’09, Valencia, Spain, April 15 - 17.

[2] C. Ghiță, Electromechanical converters, Vol. 1 and 2, ICPE Press, Bucharest, 1999.

[3] C. Bălă, "Electrical machines", EDP Press, Bucharest, 1982.

[4] Al. Fransua, R. Măgureanu, "Electrical Machines and Drive Systems”, Technical Press, Oxford, 1984.

[5] C. GHIȚĂ, “The simultaneous use of synchronous machine as motoring and compensating", International Symposium Universitaria ROPET 2001, Petroşani, ROMANIA, 18 - 20 oct. 2001, Vol. 4, Electrical Engineering, pp. 211 - 214.

[6] F. Ionescu, S. Nițu, D. Floricău, D. Fodor, Power electronics - Static Converters, Bucharest, Technical Press 1998 ;

[7] www.semikron.com / Power Semiconductor Catalog. 